\title{
Folic Acid Metabolic Process
}

National Cancer Institute

\section{Source}

National Cancer Institute. Folic Acid Metabolic Process. NCI Thesaurus. Code C19956.

Metabolic processes that utilize folic acid (as tetrahydrofolate) as a carbon unit donor. 